\title{
HLA-DMA Gene
}

National Cancer Institute

\section{Source}

National Cancer Institute. HLA-DMA Gene. NCI Thesaurus. Code C150071.

This gene plays a role in removing the class II-associated invariant chain peptide (CLIP) from newly synthesized class II major histocompatibility protein complexes. 